EL CONOCIMIENTO DE LA GRAMÁTICA DE LA LENGUA ESPAÑOLA COMO FACTOR CONDICIONANTE PARA EL APRENDIZAJE DEL IDIOMA INGLÉS

\title{
EL CONOCIMIENTO DE LA GRAMÁTICA DE LA LENGUA ESPAÑOLA COMO FACTOR CONDICIONANTE PARA EL APRENDIZAJE DEL IDIOMA INGLÉS.
}

Candelaria Vázquez Ramos*, Rita del Pilar Zurita Fonz**

*Doctora en Planeación Estratégica y Dirección de Tecnología. Profesora-Investigadora. División Académica de Ciencias Económico Administrativas (DACEA). Universidad Juárez Autónoma de Tabasco (UJAT).

**Maestra en Docencia. Profesora-Investigadora. Centro de Enseñanza de Lenguas Extranjeras (CELE)-UJAT.

Dirección para recibir correspondencia: cvazquezramos@hotmail.com

Fecha de recibido: 4 de noviembre de 2016

Fecha de aceptación: 13 de diciembre de 2016 
EL CONOCIMIENTO DE LA GRAMÁTICA DE LA LENGUA ESPAÑOLA COMO FACTOR CONDICIONANTE PARA EL APRENDIZAJE DEL IDIOMA INGLÉS

\section{RESUMEN}

Objetivo: Identificar en qué medida el nivel de dominio básico de conocimiento de la gramática española influye para el proceso de aprendizaje del idioma inglés como segunda lengua de los estudiantes del Centro de Enseñanza de Idiomas de la Universidad Juárez Autónoma de Tabasco.

Material y método: Para la realización de la investigación se generó un modelo correspondiente a un diseño cuasiexperimental que se aplicó en dos grupos de estudiantes denominados "A" y "B", los cuales arrojaron un total de 36 informantes. El fundamento teórico que soporta la investigación es el constructivismo, en función de tres categorías de análisis desarrolladas por Lev Vigotsky (2007): el conocimiento previo, el aprendizaje significativo y el proceso de integración-asimilación del conocimiento.

Para determinar el nivel de conocimiento de gramática española de los alumnos sujetos de la investigación se realizó un examen diagnóstico al comienzo del curso, así como seis exámenes que definieron su aprovechamiento respecto al aprendizaje del idioma inglés.

Resultados: Para la comprobación de la hipótesis se estudió la relación existente entre las variables de estudio propuestas, a saber: el conocimiento previo de la gramática española como factor que condiciona el aprendizaje del idioma inglés en tanto segunda lengua.

A partir de un análisis de correlación lineal, se obtuvieron resultados que mostraron que la dependencia entre las dos variables del estudio es muy alta, toda vez que en el grupo "A" este índice fue de 0.67 y en el "B" de 0.71 , consignándose una variación mínima entre ambos del orden de 0.04 , situación que confirmó la validez de la hipótesis sugerida para la investigación.

Conclusiones: A partir de los resultados obtenidos en la investigación, se encontró que el conocimiento previo de la gramática española si fue un factor que incide en el aprendizaje del idioma inglés como segunda lengua. La aportación de este estudio es servir de base para proponer que se diseñe un curso de gramática española para los estudiantes del primer semestre del curso de inglés del Centro de Enseñanza de Lenguas Extranjeras (CELE), que podría hacerse extensivo a los estudiantes de otros semestres del idioma inglés e incluso para estudiantes de otros idiomas, para una mejor asimilación y aprovechamiento en el proceso de enseñanza aprendizaje.

Palabras clave: Aprendizaje. Factores. Constructivismo. Conocimiento. Integración. 
EL CONOCIMIENTO DE LA GRAMÁTICA DE LA LENGUA ESPAÑOLA COMO FACTOR CONDICIONANTE PARA EL APRENDIZAJE DEL IDIOMA INGLÉS

\section{ABSTRACT}

Objective: To identify to what extent the basic level of domain knowledge of Spanish grammar influences to the process of learning English as a second language students Language Training Center of the Juarez Autonomous University of Tabasco.

Material and methods: To carry out research corresponding to a quasi-experimental design model was applied to two groups of students called A and B, which yielded a total of 36 informants was generated. The theoretical foundation that supports research is constructivism, based on three categories of analysis developed by Lev Vygotsky (2007): previous knowledge, meaningful learning and the integration process of knowledge assimilation.

To determine the level of knowledge of Spanish grammar student research subjects diagnosed early in the course examination was performed, and six tests that defined their achievement toward learning English.

Results: To test the hypothesis the relationship between the variables of study proposals were studied, namely prior knowledge of Spanish grammar as a factor determining learning English as a second language.

From a linear correlation analysis, results showed that the dependence between the two variables of the study is very high, since in group $A$ this index was 0.67 and $B 0.71$ were obtained, stating minimal variation between the order of 0.04 , a situation confirmed the validity of the suggested research hypothesis.

Conclusions: From the results obtained in the investigation, it was found that prior knowledge of Spanish grammar if it was a factor that affects learning English as a second language. The contribution of this study is to provide a basis for proposing a course of Spanish grammar is designed for students in the first semester of English Course Center Teaching of Foreign Languages, which could be extended to students from other semesters of English and even students from other languages, for better assimilation and utilization in the teaching-learning process.

Keywords: Learning. Factors. Constructivism. Knowledge. Integration. 
EL CONOCIMIENTO DE LA GRAMÁTICA DE LA LENGUA ESPAÑOLA COMO FACTOR CONDICIONANTE PARA EL APRENDIZAJE DEL IDIOMA INGLÉS

\section{INTRODUCCIÓN}

Este trabajo representa una aproximación inicial encaminada a esclarecer una inquietud surgida en la práctica docente en el ámbito de la enseñanza del idioma inglés, a saber: ¿El conocimiento que los alumnos poseen acerca de la gramática del español, facilita el aprendizaje del inglés como segunda lengua, permitiendo ampliar las posibilidades de llegar a dominarlo y desarrollar las distintas habilidades que ello implica?

La ruta que se ha trazado para resolver tal inquietud, conlleva a plantear, examinar y evaluar las características de la relación que se da entre las dos variables de estudio (conocimiento de gramática española/aprendizaje del inglés); los tramos que componen la ruta están delineados por cada uno de los puntos que integran la investigación.

Se describe el problema, definiendo las condiciones espacio-temporales de la investigación. Sus propósitos y alcances, así como las herramientas teóricas que se consideran pertinentes para el tratamiento del problema. El enfoque teórico al que se recurre es el denominado como constructivismo, específicamente los planteamientos de David P. Ausubel acerca del aprendizaje significativo.

Se realiza reseña de los procesos de las principales teorías de aprendizaje, sin tratar de abarcar la historia del problema como un continuo, sino solo compendiar las más significativas con relación al enfoque teórico seleccionado.

Del mismo modo se consideran las teorías acerca de la adquisición del lenguaje, de una manera genérica, así como las corrientes más representativas sobre la adquisición de una segunda lengua.

Se presentan las principales corrientes de estudio acerca de la enseñanza específica del idioma inglés y se proporciona un resumen de los conceptos esenciales de la gramática española, centrándose en aquellos aspectos sintácticos que son relevantes en ambas lenguas.

Se identificó que el conocimiento de conceptos gramaticales del idioma español que poseían muchos de los alumnos al inicio del curso del idioma inglés les sirvió de sustento para compararlos con los conceptos de este idioma y fue un factor que les permitió mayor 
EL CONOCIMIENTO DE LA GRAMÁTICA DE LA LENGUA ESPAÑOLA COMO FACTOR CONDICIONANTE PARA EL APRENDIZAJE DEL IDIOMA INGLÉS

aprovechamiento en su estudio, es decir, que el poseer una base conceptual de la gramática de su idioma materno les facilitó el reconocer similitudes y diferencias entre conceptos gramaticales de ambos idiomas.

\section{Desarrollo}

\section{Problemática del estudio}

El interés para realizar esta investigación nació de la experiencia profesional que nos permitió una serie de situaciones que surgen durante el proceso de aprendizaje del idioma inglés y que se relacionan directamente con el conocimiento de la gramática de la lengua española, así como el efecto que produce la carencia del mismo en dicho proceso.

Lo anterior, en virtud de que la falta del referente de la gramática de su lengua materna, provoca que el estudiante no puede hacer analogías o encontrar similitudes o diferencias entre ésta y la lengua que está aprendiendo, situación distinta a la que presentan aquellos estudiantes que si poseen conocimiento de la gramática española.

Ejemplos de esta dificultad se presentan cuando al explicar algún punto gramatical, utilizando términos como adjetivo, pronombre, verbo, los estudiantes desconocen a qué se refieren estos conceptos.

Por ello, consideramos de suma importancia identificar en qué medida el conocimiento previo de la gramática de la lengua española es un factor que incide para facilitar el proceso de aprendizaje del idioma inglés en un entorno académico. Con esa finalidad, se plantearon las siguientes cuestiones a dilucidar:

1. Relevancia del conocimiento de la gramática española como condicionante para el proceso de aprendizaje del idioma inglés como segunda lengua.

2. Determinar el nivel de conocimiento sobre gramática española que presentan los alumnos al inicio del primer semestre de inglés del curso básico del Centro de Enseñanza de Idiomas.

3. Reconocer los factores que condicionan el proceso de aprendizaje del idioma inglés como segunda lengua.

4. Definir formas para la evaluación de la interdependencia entre el conocimiento de la gramática española y la capacidad de aprendizaje del idioma inglés como segunda lengua.

5. Diseñar una propuesta pedagógica que permita mejorar el proceso de aprendizaje del inglés con base en el conocimiento previo de la gramática española. 
EL CONOCIMIENTO DE LA GRAMÁTICA DE LA LENGUA ESPAÑOLA COMO FACTOR CONDICIONANTE PARA EL APRENDIZAJE DEL IDIOMA INGLÉS

\section{Justificación}

En la actualidad, el aprendizaje de otro $u$ otros idiomas se ha convertido en un requerimiento esencial e impostergable en las diversas áreas de estudio y actividades profesionales que desarrollan las personas, y siendo, además, el idioma inglés el de uso generalizado en la mayoría de los campos del quehacer humano, se hace prioritario que los docentes dedicados a la enseñanza de idiomas estemos atentos a las necesidades de nuestros alumnos.

En ese sentido, es menester interesarnos y dedicarnos a la investigación que permita mejorar el proceso de aprendizaje de los idiomas, en particular, el idioma inglés, con la finalidad de encontrar herramientas para hacer ese proceso más accesible, eficaz y agradable.

De tal manera, la premisa para este trabajo fue identificar en qué medida el conocimiento de la gramática de la lengua española facilita a los estudiantes el proceso de aprendizaje del idioma inglés, con el propósito de que los resultados de la investigación sean la base para diseñar y proponer opciones metodológicas adecuadas y aplicarlas en las aulas donde se enseña dicho idioma en nuestra institución en beneficio de nuestros alumnos.

\section{Marco teórico}

Para llevar a cabo el estudio, se consideraron los postulados de la teoría constructivista del aprendizaje, los cuales sostienen que la interacción entre el objeto de estudio y el sujeto cognoscente constituye la esencia del proceso de aprendizaje.

En este enfoque se visualiza al individuo desde los aspectos cognoscitivos, sociales y afectivos, concibiéndolo como una construcción propia que se conforma día a día como producto del ambiente y de sus disposiciones internas.

Por lo tanto, el conocimiento no es una copia de la realidad, sino una construcción que realiza el ser humano a partir de los esquemas que ya posee y de aquellos que va construyendo en su relación con el medio que lo rodea.

En este contexto se plantea el término de aprendizaje significativo, el cual se genera cuando el sujeto, en su carácter de constructor de su propio conocimiento, configura y pondera los conceptos a aprender, en función de la estructura conceptual que ya posee, estableciendo relaciones de subordinación, coordinación o supraordinación entre unos y otros. 
EL CONOCIMIENTO DE LA GRAMÁTICA DE LA LENGUA ESPAÑOLA COMO FACTOR CONDICIONANTE PARA EL APRENDIZAJE DEL IDIOMA INGLÉS

Con base en tales argumentos, se planteó el supuesto de investigación que afirma que:

"Los alumnos que poseen conocimientos de la gramática de su idioma materno, en este caso el español, demuestran una mayor capacidad y facilidad de aprendizaje [del idioma inglés], puesto que entienden mejor los conceptos y estructuras gramaticales que están aprendiendo, adquiriéndolo con mayor facilidad que aquellos alumnos que carecen del conocimiento básico de la gramática de su propio idioma".

\section{METODOLOGÍA}

Se trata de una investigación de enfoque cuantitativo, de tipo descriptivo ya que con base en la recolección de la información se pretende determinar el nivel de conocimiento de la gramática de la lengua española que presenta cada grupo y en función de ello establecer cómo incide dicho factor en el aprendizaje del idioma inglés como segunda lengua.

El diseño es longitudinal ya que los datos se recolectaron en diversos momentos por medio de los instrumentos de investigación que se detallan más adelante.

El modelo de cuasiexperimento utilizado para la investigación es el que corresponde, conforme a la clasificación de Donald Campbell (2005:110), al diseño de ciclo institucional recurrente, mismo que se entiende como aquel que presenta de forma cíclica a cada nuevo grupo de participantes cierto aspecto de un proceso institucional en cual la variable experimental resulta recurrente y se presenta constantemente a cada nuevo grupo de participantes (Campbell, 2005. pp. 111-112).

Las categorías de análisis que fueron utilizadas para validar la hipótesis de a investigación fueron:

Categoría 1. Conocimiento previo o estructura cognitiva: consiste en los esquemas o representaciones que el sujeto cognoscente ya posee.

Categoría 2. Aprendizaje significativo: "el aprendizaje significativo es aquel que conduce a la creación de estructuras de conocimiento mediante la relación sustantiva entre la nueva información y las ideas previas de los estudiantes", (Díaz Barriga y Hernández Rojas, 2005:39).

Categoría 3. Proceso de integración-asimilación del conocimiento: Postula que una vez que el sujeto se encuentra en disposición para aprender, la relación con los esquemas nuevos se verifica a partir de los elementos relevantes y sustanciales que subyacen en el conocimiento ya existente. 
EL CONOCIMIENTO DE LA GRAMÁTICA DE LA LENGUA ESPAÑOLA COMO FACTOR CONDICIONANTE PARA EL APRENDIZAJE DEL IDIOMA INGLÉS

Así pues, el conocimiento de la gramática española constituye el conocimiento previo que poseen los alumnos, mientras que la temática que compone el programa de primer semestre de inglés representa el conjunto de esquemas o información nueva a la que se enfrenta el estudiante, quien realiza durante el curso el proceso de organización, transformación e interpretación de lo nuevo, ponderando aquello que resulta significativo, en función de los esquemas de lo conocido con anterioridad.

La selección de la muestra estuvo conformada por dos grupos de estudiantes de primer semestre de inglés del CELE de la UJAT denominados: "A" y "B", mismos que representaron un caso típico, toda vez que configuraban las condiciones delineadas por la situación a estudiar.

Como variable independiente del estudio se definió el nivel de conocimiento previo de la gramática española, siendo la variable independiente el grado de aprovechamiento en el aprendizaje del idioma inglés.

Asimismo, se utilizaron como instrumentos de investigación los siguientes:

Un examen diagnóstico de gramática española a cada grupo, aplicado al comienzo del curso respectivo, con el propósito de establecer el nivel de conocimiento de la gramática española que poseían los informantes previos a la aplicación del modelo.

Para determinar el grado de aprovechamiento en el aprendizaje del idioma inglés de cada uno de los informantes, se verificaron tres exámenes parciales, dos exámenes de medio semestre y un examen final.

\section{RESULTADOS}

En primer lugar, se muestran los resultados obtenidos por los sujetos de estudio del Grupo "A", referentes a: examen diagnóstico y exámenes de inglés (para un mejor manejo de la información se consignan solamente números enteros). 
EL CONOCIMIENTO DE LA GRAMÁTICA DE LA LENGUA ESPAÑOLA COMO FACTOR CONDICIONANTE PARA EL APRENDIZAJE DEL IDIOMA INGLÉS

Tabla 1

Resultados obtenidos de los estudiantes de estudio del Grupo "A"

\begin{tabular}{ccc}
\hline Sujeto & Examen diagnóstico & Calificación inglés \\
\hline 1 & 10 & 7 \\
2 & 9 & 6 \\
3 & 10 & 9 \\
4 & 8 & 5 \\
5 & 10 & 7 \\
6 & 10 & 9 \\
7 & 10 & 8 \\
8 & 10 & 9 \\
9 & 10 & 9 \\
10 & 10 & 9 \\
11 & 10 & 8 \\
12 & 10 & 8 \\
13 & 10 & 9 \\
14 & 10 & 9 \\
15 & 6 & 6 \\
16 & 10 & 7 \\
17 & 10 & 8 \\
\hline
\end{tabular}

Fuente: Elaboración propia.

En este grupo, el promedio de las calificaciones del examen diagnóstico fue superior a 9 y el promedio de las calificaciones de inglés es 7.8.

El total de los sujetos aprobó el examen diagnóstico, en términos prácticos 8 de cada 10 de ellos obtuvo una calificación igual o superior a 8.

Más del 90\% de los alumnos aprobaron el curso de inglés y, en números redondos, 6 de cada 10 de ellos obtuvieron calificación igual o superior a 8.

El índice de correlación lineal entre ambas calificaciones es de 0.67 , el cual se obtuvo empleando la siguiente fórmula: 
EL CONOCIMIENTO DE LA GRAMÁTICA DE LA LENGUA ESPAÑOLA COMO FACTOR CONDICIONANTE PARA EL APRENDIZAJE DEL IDIOMA INGLÉS

$$
r=\frac{\sum_{1}^{17}(x-\bar{x})(y-\bar{y})}{\sqrt{\sum_{1}^{17}(x-\bar{x})^{2}} \sqrt{\sum_{1}^{17}(y-\bar{y})^{2}}}
$$

En donde:

$r=$ coeficiente de correlación lineal.

x = calificación del examen diagnóstico de gramática española.

y = calificación de inglés.

$x($ con tilde $)=$ media aritmética de las calificaciones del examen diagnóstico .

y $($ con tilde $)=$ media aritmética de las calificaciones de inglés.

El valor del coeficiente de correlación lineal implica una fuerte dependencia entre las variables (calificación del examen diagnóstico y calificación de inglés).

A continuación se muestra la gráfica de ello, el eje vertical representa las calificaciones y el eje horizontal representa a cada individuo de la muestra.

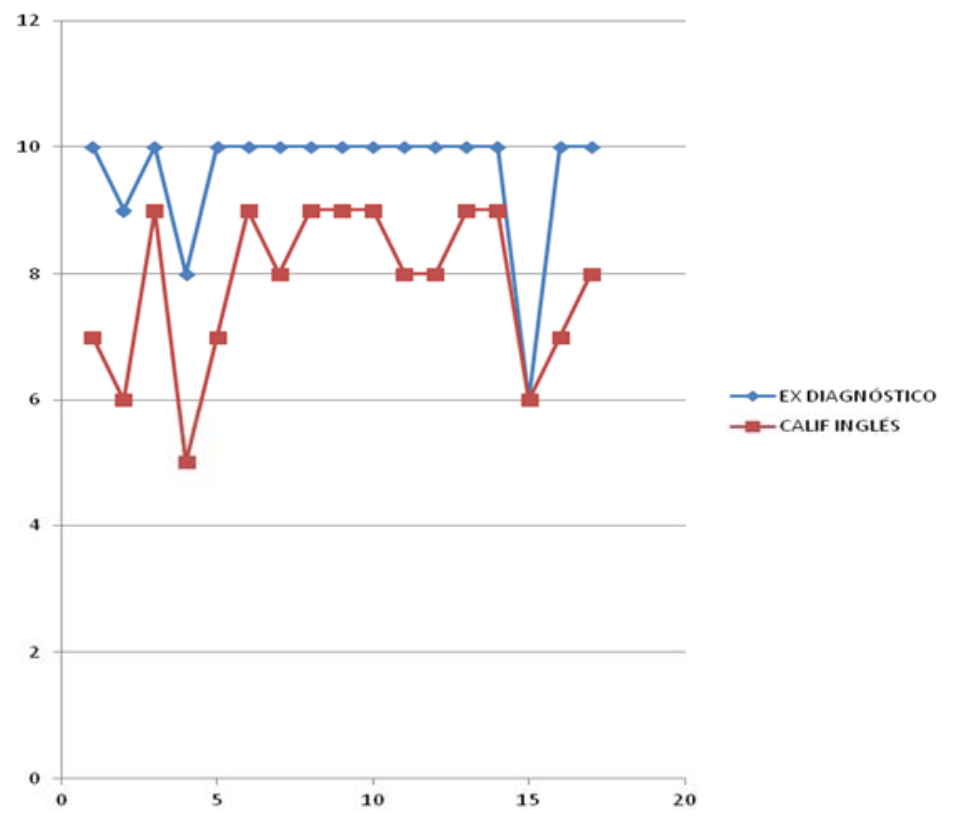

Figura 1. Gráfica de correlación lineal del Grupo "A". 
EL CONOCIMIENTO DE LA GRAMÁTICA DE LA LENGUA ESPAÑOLA COMO FACTOR CONDICIONANTE PARA EL APRENDIZAJE DEL IDIOMA INGLÉS

El análisis de correlación lineal del Grupo "A" permite hacer las siguientes observaciones respecto de la causalidad entre las variables de estudio:

14 de 17 sujetos de la muestra obtuvieron la calificación máxima (10) en la evaluación de gramática (Sujetos 1, 3, 5, 6, 7, 8, 9, 10, 11, 12, 13, 14, 16, 17).

7 de esos mismos alumnos obtuvieron la calificación máxima (9) en inglés (Sujetos 3, 6, 8, 9, $10,13,14)$.

4 de esos mismos sujetos obtuvieron la segunda calificación más alta (8) en inglés (Sujetos 7, $11,12,17)$.

Los dos sujetos que obtuvieron las calificaciones más bajas en gramática obtuvieron las dos calificaciones más bajas en inglés (Sujetos 4, 15).

A continuación, se presenta la Tabla 2, la cual contiene los resultados de los estudiantes del Grupo "B".

\section{Tabla 2}

Resultados obtenidos de los estudiantes del Grupo "B".

\begin{tabular}{ccc}
\hline SUJETO & EXAMEN DIAGNÓSTICO & CALIFICACIÓN INGLÉS \\
\hline 1 & 3 & 4 \\
2 & 3 & 3 \\
3 & 4 & 3 \\
4 & 4 & 4 \\
5 & 5 & 5 \\
6 & 5 & 8 \\
7 & 6 & 7 \\
8 & 5 & 5 \\
9 & 6 & 6 \\
10 & 6 & 3 \\
11 & 6 & 8 \\
12 & 6 & 7 \\
13 & 6 & 6 \\
14 & 6 & 5 \\
15 & 6 & 7 \\
16 & 6 & 5
\end{tabular}


EL CONOCIMIENTO DE LA GRAMÁTICA DE LA LENGUA ESPAÑOLA COMO FACTOR CONDICIONANTE PARA EL APRENDIZAJE DEL IDIOMA INGLÉS

\begin{tabular}{lll}
17 & 7 & 9 \\
18 & 7 & 7 \\
19 & 8 & 8 \\
\hline
\end{tabular}

Fuente: Elaboración propia.

El promedio de las calificaciones del examen diagnóstico en esta muestra fue inferior a 6 (5.58) y el promedio de las calificaciones de inglés también es inferior a 6 (5.79).

Poco más de la mitad (63.16\%) de los sujetos aprobó el examen diagnóstico, pero solamente el $5 \%$ obtuvo una calificación igual o superior a 8.

Prácticamente la mitad (52.63\%) de los alumnos aprobaron el curso de inglés y, en números redondos, 2 de cada 10 de ellos obtuvieron calificación igual o superior a 8.

El índice de correlación lineal entre ambas calificaciones es de 0.71 , el cual se obtuvo empleando la siguiente fórmula:

$$
r=\frac{\sum_{1}^{19}(x-\bar{x})(y-\bar{y})}{\sqrt{\sum_{1}^{19}(x-\bar{x})^{2}} \sqrt{\sum_{1}^{17}(y-\bar{y})^{2}}}
$$

En donde:

$r=$ coeficiente de correlación lineal.

x = calificación del examen diagnóstico de gramática española.

y = calificación de inglés.

$x($ con tilde $)=$ media aritmética de las calificaciones del examen diagnóstico.

y $($ con tilde $)=$ media aritmética de las calificaciones de inglés.

Lo cual implica una fuerte dependencia entre variables (calificación del examen diagnóstico y calificación de inglés). 
EL CONOCIMIENTO DE LA GRAMÁTICA DE LA LENGUA ESPAÑOLA COMO FACTOR CONDICIONANTE PARA EL APRENDIZAJE DEL IDIOMA INGLÉS

A continuación, se muestra la gráfica de ello, el eje vertical representa las calificaciones y el eje horizontal representa a cada individuo de la muestra.

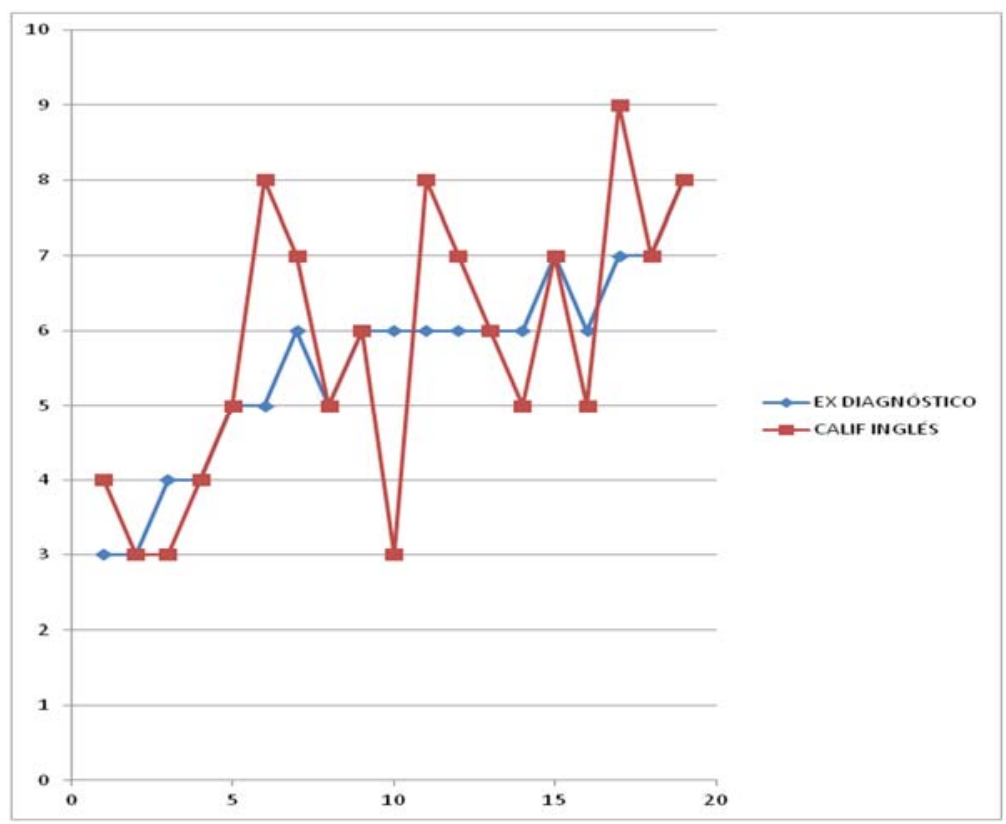

Figura 2. Gráfica de correlación lineal del Grupo "B".

El análisis de correlación lineal del Grupo B permite hacer las siguientes observaciones respecto de la causalidad entre las variables de estudio:

9 de 12 de los sujetos con calificaciones aprobatorias en gramática, aprueban también en la evaluación de inglés (Sujetos 7, 9, 11, 12, 13,15, 17, 18,19).

El sujeto con la calificación más alta en inglés (Sujeto 17) es uno de los tres con la segunda mejor calificación en gramática, los cuales fueron superados solo por el sujeto 19 en dicha evaluación.

El sujeto 19, con la mejor calificación en gramática, fue uno de los tres con la segunda mejor calificación en inglés, siendo a su vez superado únicamente por el Sujeto 17 en este rubro.

9 de los 19 sujetos del Grupo "B" presentan calificaciones coincidentes tanto en gramática como en inglés (Sujetos 2, 4, 5, 8, 9, 13, 15, 18, 19). 
EL CONOCIMIENTO DE LA GRAMÁTICA DE LA LENGUA ESPAÑOLA COMO FACTOR CONDICIONANTE PARA EL APRENDIZAJE DEL IDIOMA INGLÉS

\section{Hallazgos}

Por un lado, se encontró que en el Grupo "A", los diecisiete sujetos que conformaron la muestra aprobaron el examen diagnóstico acerca del conocimiento de la gramática del idioma español; de ellos, catorce obtuvieron 10 de calificación, uno obtuvo 9, uno obtuvo 8 y uno obtuvo 6.

En tanto que en la calificación acerca del aprovechamiento en el aprendizaje del idioma inglés, siete de ellos aprobaron con 9, cuatro con 8, tres con 7, dos obtuvieron 6 y uno obtuvo 5. Hay que mencionar que de los sujetos que alcanzaron calificación de 10 en el examen diagnóstico acerca del conocimiento de la gramática del idioma español, no todos lograron 9 en el aprovechamiento en el aprendizaje del idioma inglés que fue la calificación más alta obtenida en este rubro.

A continuación, se desglosan los resultados obtenidos por los diecisiete sujetos, mencionando la calificación del examen diagnóstico y la del aprovechamiento en el aprendizaje del idioma inglés.

Siete sujetos que aprobaron con 10 de calificación el examen diagnóstico de español obtuvieron 9 de calificación en el aprovechamiento en el aprendizaje del idioma inglés que fue la más alta.

Cuatro sujetos que alcanzaron 10 en el examen diagnóstico obtuvieron 8 en el aprovechamiento en el aprendizaje del idioma inglés. Tres sujetos que alcanzaron 10 en el examen diagnóstico obtuvieron 7 en el aprovechamiento en el aprendizaje del idioma inglés.

A partir del análisis de ambos resultados se presentan las siguientes observaciones:

- Catorce de diecisiete sujetos del Grupo A obtuvieron la calificación de 10 en el examen diagnóstico (sujetos 1, 3, 5, 6, 7, 10, 11, 12, 13, 14, 16, 17).

- Siete de ellos lograron la calificación máxima (9) de aprovechamiento de inglés (sujetos 3, 6, $8,9,10,13,14)$.

- Cuatro de esos 14 sujetos que alcanzaron 10 en el examen diagnóstico obtuvieron la segunda calificación más alta (8) en el aprovechamiento del inglés (sujetos 7, 11, 12, 17).

- Los dos sujetos que obtuvieron las calificaciones más bajas en el examen diagnóstico (8 y 6), tuvieron las dos calificaciones más bajas (5 y 6) en el aprovechamiento del inglés (sujetos 4,15).

Al considerar el índice de correlación lineal entre ambas calificaciones, el cual es de 0.67, puede afirmarse que en el Grupo "A", la hipótesis que se plantea en este trabajo acerca de "el conocimiento de la gramática del español como factor condicionante para el aprendizaje del 
EL CONOCIMIENTO DE LA GRAMÁTICA DE LA LENGUA ESPAÑOLA COMO FACTOR CONDICIONANTE PARA EL APRENDIZAJE DEL IDIOMA INGLÉS

idioma inglés" implica una fuerte dependencia entre las variables conocimiento de la gramática del español y aprendizaje del idioma inglés.

Por lo que hace al Grupo "B", a continuación se desglosan las calificaciones obtenidas en ambas evaluaciones, así como las implicaciones extraídas.

En este grupo hubo diecinueve sujetos, de los cuales doce obtuvieron calificación aprobatoria en el examen diagnóstico, de la forma que sigue: un informante obtuvo 8 (sujeto 19); tres sujetos 7 (sujetos 15, 17, 18); finalmente, ocho sujetos obtuvieron 6 (sujetos 7, 9, 10, 11, 12, 13, $14,16)$.

De los sujetos que sacaron calificación reprobatoria (menos de 6) en el examen diagnóstico, la información se desglosa como sigue: tres sujetos obtuvieron 5 de calificación (sujetos 5, 6, 8); dos sujetos 4 de calificación (sujetos 3, 4); dos sujetos 3 de calificación (sujetos 1,2).

En cuanto a los resultados en el aprovechamiento en el aprendizaje del idioma inglés, los mismos se dieron de la siguiente manera:

Un informante alcanzó 9 (calificación más alta de aprovechamiento en el aprendizaje del inglés) y había obtenido 6 en el examen diagnóstico (sujeto 17). Tres sujetos lograron 8, uno de ellos había alcanzado 6 en el examen diagnóstico (sujeto 11), otro había obtenido 5 en el examen diagnóstico (sujeto 6) y el otro 8 en el examen diagnóstico (sujeto 19).

Cuatro sujetos obtuvieron 7 en el examen de aprovechamiento en el aprendizaje el idioma inglés, cuales: dos habían alcanzado 7 en el examen diagnóstico (sujetos 15,18), mientras que los otros dos habían obtenido 6 (sujetos 7, 12).

Dos sujetos alcanzaron 6 en el aprovechamiento y habían obtenido 6 en el examen diagnóstico (sujetos 9, 13).

Cuatro sujetos obtuvieron 5 en el aprovechamiento de inglés de los cuales, dos habían calificado con 6 en el examen diagnóstico (sujetos 14, 16) y dos habían calificado con 5 en el examen diagnóstico (sujetos 5, 8).

Dos sujetos obtuvieron 4 en el aprovechamiento de inglés uno había alcanzado 4 en el examen diagnóstico (sujeto 4) y el otro había calificado con 3 en el examen diagnóstico (sujeto 1). 
EL CONOCIMIENTO DE LA GRAMÁTICA DE LA LENGUA ESPAÑOLA COMO FACTOR CONDICIONANTE PARA EL APRENDIZAJE DEL IDIOMA INGLÉS

Tres sujetos obtuvieron 3 en el aprovechamiento de inglés, de ellos, uno había obtenido 6 en el examen diagnóstico (sujeto 10), otro más había obtenido 4 en el examen diagnóstico (sujeto 3) y el restante había obtenido 3 en el examen diagnóstico (sujeto 2).

De acuerdo a estos resultados y tomando en consideración el índice de correlación lineal entre ambas calificaciones, el cual es de 0.71 , puede decirse que existe una fuerte dependencia entre las variables conocimiento de la gramática del español y el aprendizaje del idioma inglés.

El índice de correlación lineal de ambos grupos (0.67 y 0.71) difiere en una magnitud mínima (0.04) por lo que, en el contexto de la muestra seleccionada, la relación de causalidad o dependencia entre las variables debe calificarse como muy alta.

\section{CONCLUSIONES}

La idea de hacer ésta investigación, nace al reconocer como una problemática que, el conocimiento previo de la gramática española, que tienen los estudiantes al inicio del curso incide en el proceso de aprendizaje del idioma inglés como segunda lengua, en los alumnos del primer semestre del curso de inglés del Centro de Enseñanza de idiomas de la Universidad Juárez Autónoma de Tabasco.

Lo anterior debido a que es necesario que los alumnos tengan los conocimientos básicos acerca de los conceptos gramaticales que se manejan en el programa de dicho curso, lo cual les sirve de sustento para adquirir los conocimientos acerca de la lengua inglesa a través de reconocer elementos lingüísticos y gramaticales en razón que todas las lenguas poseen elementos gramaticales que pueden ser iguales, similares o diferentes a los de la gramática del español.

Al considerar lo anterior, el objetivo general para esta investigación, fue identificar si el conocimiento previo de la gramática del idioma español facilita a los estudiantes el proceso de aprendizaje de la lengua inglesa, para lo cual se aplicó un examen diagnóstico acerca del conocimiento de la gramática española al inicio del curso, así mismo, exámenes de aprovechamiento del idioma inglés a lo largo de éste para obtener calificaciones de ambos rubros mediante los cuales se pudo establecer una correlación. 
EL CONOCIMIENTO DE LA GRAMÁTICA DE LA LENGUA ESPAÑOLA COMO FACTOR CONDICIONANTE PARA EL APRENDIZAJE DEL IDIOMA INGLÉS

A partir de los resultados obtenidos en la investigación, se encontró que el conocimiento previo de la gramática española si fue un factor que incide en el aprendizaje del idioma inglés como segunda lengua. La aportación de este estudio es servir de base para proponer que se diseñe un curso de gramática española para los estudiantes del primer semestre del curso de inglés del CELE, que podría hacerse extensivo a los estudiantes de otros semestres del idioma inglés e incluso para estudiantes de otros idiomas, para una mejor asimilación y aprovechamiento en el proceso de enseñanza aprendizaje.

\section{REFERENCIAS BIBLIOGRÁFICAS}

Brooks, J. y. (1993). In search of understanding the case for constructivist classrooms. Brooks, J.G. y Brooks, G. (1993). In search of understanding. The case for constructivist classrooms. Virginia, VA.: ASCD.: Virginia, VA:ASCD.

Campbell, D. D. (1995). Diseños experimentales y cuasiexperimentales en la investigación social. Buenos Aires, Argentina: Amorrortu, Editores .

Díaz-Barriga Arceo, F. y. (2005). Estrategías docentes para un aprendizaje significativo. Una interpretación constructivista. México: Mc Graw Hill.

Hernández Sampieri, R. F. (2002). Metodología de la investigación . México: Mc Graw Hill.

Lightbown, P. M. (2008). How languages area learned. U.S.A. : Oxford University Press.

Vigotsky, Lev. (2007). Pensamiento y habla (1ª ed.). Buenos Aires: Colihue Clásica.

White, L. (2003). Second language acquisition and universal grammar. Cambridge: Cambridge University Press.

Yule, G. (1990). The study of language. An introduction. Cambridge, Great Britain: Cambridge University Press. 